\title{
IDENTIFICATION OF KURARINONE BY LC/MS AND INVESTIGATION OF ITS THERMAL STABILITY
}

\author{
PENGFEI LIU ${ }^{a, b}$, TIANSHENG DENG ${ }^{a}$, CUIPING YE ${ }^{c}$, ZHANGFENG QIN ${ }^{a}$, XIANGLIN HOU $^{a, I^{*}}, J_{I A N G U O ~ W A N G}{ }^{a}$ \\ ${ }^{a}$ State Key Laboratory of Coal Conversion, Institute of Coal Chemistry, Chinese Academy of Sciences, P.O. Box 165, Taiyuan, Shanxi 030001, PR China \\ ${ }^{b}$ Graduate University of Chinese Academy of Sciences, Beijing 100039, PR China \\ ${ }^{c}$ Key Laboratory of Coal Science and Technology of Ministry of Education and Shanxi Province, Taiyuan University of Technology, Taiyuan,
} Shanxi 030024, PR China

(Received 7 July 2008 - Accepted 17 November 2008)

\begin{abstract}
A herbal ingredient kurarinone (7, 2', 4'-trihydroxy-8-lavandulyl-5-methoxy flavanone) was isolated from the roots of sophora flavescens Ait. by ultrasonic extraction, which is one of traditional Chinese medicine (TCM) materials. A reliable high performance liquid chromatography with mass spectrometry (HPLCMS) method was used for determination of kurarinone. C18 column $(150 \times 4.6 \mathrm{~mm}, 5 \mu \mathrm{m})$ and methanol-water mobile phase $(70: 30$, by volume $)$ were used in the chromatographic separation of active components from the herb. HPLC-ESI-MS (UV, $288 \mathrm{~nm} ; \mathrm{m} / \mathrm{z}, 437.5$ [M-H]) was adopted for kurarinone identification. Kurarinone with purity of $95.5 \%$ was obtained by silica gel column followed by reverse-phase column chromatography. The thermal stability of kurarinone was investigated, which illustrates that kurarinone was stable below $25^{\circ} \mathrm{C}$ in $48 \mathrm{~h}$, but a degradation of $25.54 \%$ was observed at $80{ }^{\circ} \mathrm{C}$ for $2 \mathrm{~h}$.
\end{abstract}

Keywords: Sophora flavescens Ait; Leguminosae; Flavonoids; Kurarinone; HPLC-ESI-MS; Ultrasonic extraction; Thermal stability.

\section{INTRODUTION}

There has been growing interest over the past 20 years in the natural medications because of their low toxicity and effective therapeutical performance. Increasing evidence illustrates that flavanones are beneficial to human health. The roots of sophora flavescens Ait. are used as traditional Chinese medicine (TCM) material effective in antipyretic, analgesic, anthelmintic and stomachic ${ }^{1}$. Kurarinone (7, 2', 4'-trihydroxy-8-lavandulyl-5methoxy flavanone, Fig. 1), as a natural pigment derivative from the herb $S$. flavescens ${ }^{2}$, has been proved to be effective on anti cancer, anti androgenic ${ }^{3}$, anti atherosclerotic $\mathrm{c}^{4}$, and inhibition of tyrosinase ${ }^{5}$ in recent years.

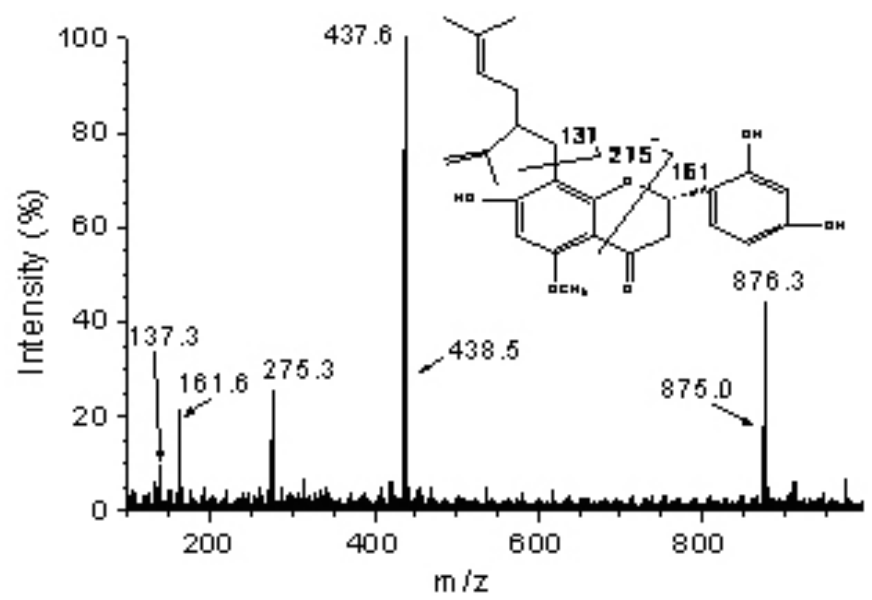

Fig. 1. Full scan mass spectrum of kurarinone and its chemical structure (molecular weight 438).

Techniques of maceration and hot extraction in Soxhlet apparatus are widely used to obtain plant extracts. However, the herbal ingredient may be degraded for long-duration heat treatment $t^{6}$. The efficiency of extraction is influenced by solvent type, solvent quantity, extraction temperature, duration and cycles ${ }^{7}$. Ultrasonic can improve the rate, selectivity and yield in extracting active components ${ }^{8}$. A reverse-phase liquid chromatography (LC) with methanol-water $(70: 30, \mathrm{v} / \mathrm{v})$ as mobile phase was used to isolate kurarinone and UV at wavelength $290 \mathrm{~nm}$ was selected for its detection ${ }^{4}$. Analyses of flavonoids with ESI-MS ${ }^{\mathrm{n}}$ in $S$. flavescens have been reported by a paper ${ }^{9}$ which gives the formulae of fracture mode of flavonoids in S. flavescens, illustrated in Fig. 2. A total of 24 flavonoids including kurarinone are identified ${ }^{10}$, but their fragment ions are not consistent with the formulae reported in Bai's work ${ }^{9}$.

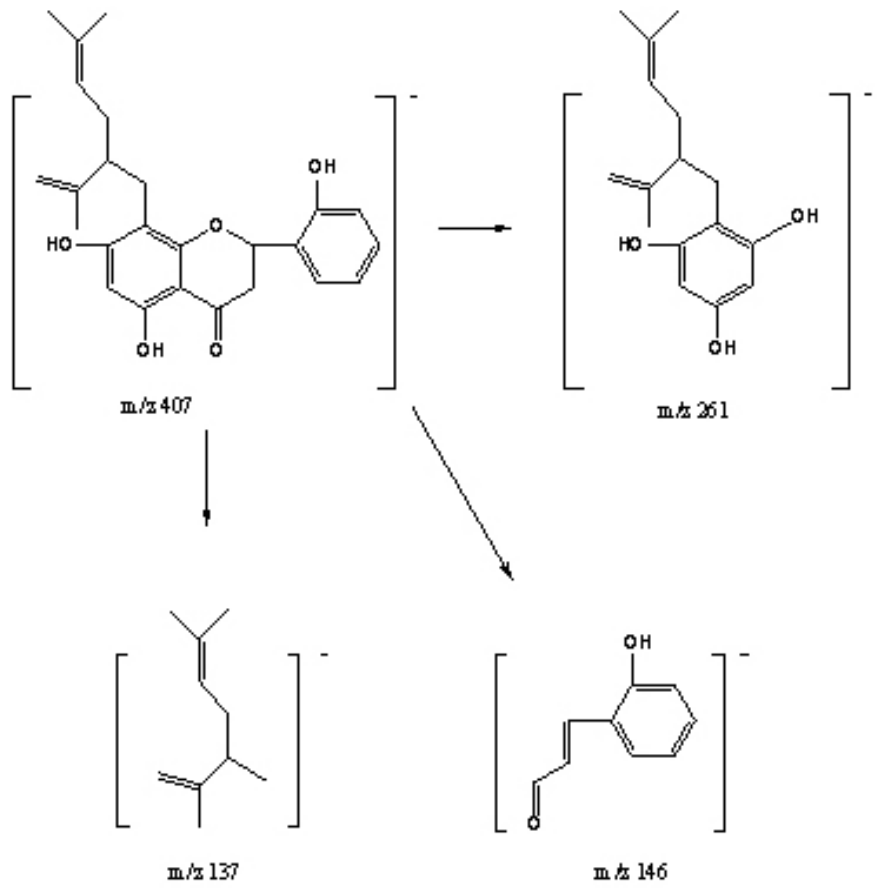

Fig. 2. The formulae of bond cleavages of flavonoid from $S$. flavescens based on Bai's work.

The major aim of this study was to verify the formulae of fracture mode of flavonoids in $S$. flavescens. In addition, the process to obtain high purity kurarinone from $S$. flavescens roots, thermal stability of kurarinone at different temperatures and the advantage of the ultrasonic extraction compared with maceration were investigated.

\section{EXPERIMENTAL}

\section{Materials}

S. flavescens radix samples (dry) purchased from the herbal store in Shuanghe Pharmaceutical Co. (Taiyuan, China) was identified by Professor J.P. Luo in Shanxi Institute of Pharmaceutical and Biological Products Controlling. Ultrasonic equipment (As2060 B) was from Autoscience Instrument Co. Ltd (Shanghai China). The authentic kurarinone was extracted from S. flavescens by us and its structure was fully characterized; the purity was above $95.5 \%$ as determined by HPLC with the UV detector at three different wavelengths ( 270 , 288 and $320 \mathrm{~nm}$ ). Vacuum desiccator was ZK-82A from Shanghai Laboratory 
Instrument General Works Co., Ltd (Shanghai China). Ethyl acetate, petroleum ether, acetone, ethanol, n-butanol and $\mathrm{n}$-hexane were of analytical grade from Tianda Chemical Reagent Co., Ltd (Tianjin China). Methanol is HPLC grade is from Tianjin Kermel Chemical Reagent Co., Ltd (Tianjin China). Re-distilled water was made in our laboratory.

\section{Sample preparation}

\section{Samples for recovery}

In order to investigate the reliability of HPLC for kurarinone detection, these assay trials were carried out. The sliced roots of S. flavescens were ground into powder and then vacuum-dried for further processing. Dried powder $(0.6$ g) added $\mathrm{x} \mathrm{ml}(\mathrm{x}=0,1,2,3)$ standard solution $(2.28 \mathrm{mg} / \mathrm{ml}$, details in 2.4 samples for liquid chromatography - mass spectrometry) was extracted with $100 \mathrm{ml}$ ethanol for $1 \mathrm{~h}$ at room temperature $\left(25^{\circ} \mathrm{C}\right)$ with ultrasonic equipment. The residue was washed with $20 \mathrm{ml}$ ethanol for 3 times. Extracts and washing solution were combined and filtered with a membrane filter of $0.45 \mu \mathrm{m}$ and added ethanol to $200 \mathrm{ml}$. Solution $(5 \mathrm{ml})$ was diluted to $25 \mathrm{ml}$ for analysis. Every group of this trial was repeated 3 times and the final added concentration of kurarinone were $0,2.28 \mu \mathrm{g} / \mathrm{ml}, 4.56 \mu \mathrm{g} / \mathrm{ml}, 6.84 \mu \mathrm{g} / \mathrm{ml}$.

\section{Samples for thermal stability}

In order to study the thermal stability $0.6 \mathrm{~g}$ dried powder was extracted with $100 \mathrm{ml}$ ethanol for $1 \mathrm{~h}$ at room temperature with ultrasonic equipment. The residue extracted by ethanol was washed by $20 \mathrm{ml}$ ethanol for 3 times. Extracts and washing solution were combined and filtered and added ethanol to $200 \mathrm{ml}$. Solution $(5 \mathrm{ml})$ was diluted to $25 \mathrm{ml}$ for analysis. This experiment was repeated 15 times.

\section{Samples for comparisons of extractive efficiency}

For comparisons of extractive efficiency between ultrasonic extraction and maceration with different parameters, four groups of samples were prepared. Dried powder $(0.6 \mathrm{~g})$ was ultrasonic extracted with $100 \mathrm{ml}$ ethanol for $1 \mathrm{~h}$ at room temperature $\left(25^{\circ} \mathrm{C}\right)$. The residue was washed by $20 \mathrm{ml}$ ethanol for 3 times. Extracts and washing solution were combined and filtered and added ethanol to $200 \mathrm{ml}$. Solution $(5 \mathrm{ml})$ was diluted to $25 \mathrm{ml}$ for analysis. The next 3 groups of trials were carried out in the following extraction conditions: ultrasonic extraction $(2 \mathrm{~h})$ and maceration $(1 \mathrm{~h}, 6 \mathrm{~h})$. All of the following trials were repeated 2 times.

\section{Samples for Liquid chromatography - mass spectrometry}

To prepare authentic standard kurarinone sample, $2 \mathrm{~kg}$ dried powder of $S$. flavescens was first extracted with 21 petroleum ether at room temperature to degrease for 3 times, each time for $2 \mathrm{~h}$. The degreased powder was vacuumdried and extracted with 51 ethanol for 3 times (each time for $40 \mathrm{~min}$ ). The extracts were combined and filtered. The filtered extract was concentrated to 11 , added 21 re-distilled water successively and placed still for $1 \mathrm{~h}$. After that, the extracts were filtered and the residue was washed with distilled water $(3 \times 0.31)$. The solid filter cake was triturated into powder. The powder was partitioned between water $(3 \times 11)$ and $n$-hexane $(3 \times 11)$, and the water phase was extracted with ethyl acetate $(3 \times 11)$ and n-butanol $(3 \times 11)$ in turn. The ethyl acetate extract $(20 \mathrm{~g})$ was subjected to silica gel column chromatography (60-100 mesh, $0.9 \mathrm{~kg})$ with a gradient of acetone/ethanol $(50: 1,30: 1,20: 1$, $10: 1,7: 1, \mathrm{v} / \mathrm{v}$, each 11$)$ as mobile phase, and 5 fractions were washed down. Fraction 4 (10:1, $2 \mathrm{~g})$ was rechromatographed over reversed phase column chromatography $(50 \mathrm{~g}$, YMC-Gel C18, $50 \mu \mathrm{m})$, eluted with methanol/water (50:50, 60:40, 70:30, 85:15, 90:10, 100:0, each $200 \mathrm{ml})$. Yellow amorphous powder (fraction F-4-3, 70:30, $0.3 \mathrm{~g}$ ) was prepared and then recrystallized in methanol/water $\left(80: 20,20 \mathrm{ml}, 5{ }^{\circ} \mathrm{C}\right)$; a pale yellow powder $(0.1 \mathrm{~g})$ was then obtained. $5 \mathrm{mg}$ standard sample (named $\beta$ ) was then dissolved in $50 \mathrm{ml}$ ethanol for HPLC-MS analysis.

Standard sample $22.6 \mathrm{mg}$ was dissolved in $100 \mathrm{ml}$ ethanol, and the solution was diluted to $2.28,4.56,9.12,18.24,22.8 \mu \mathrm{g} / \mathrm{ml}$ to establish a standard curve, $\mathrm{R}^{2}=0.9985$. Every concentration of sample was in this range.

\section{HPLC analysis}

A high performance liquid chromatograph (HPLC) coupled with a UV-vis multi-wave-length detector (LC-10ATVP Shimadzu) was used. The separation was achieved on a Shimadzu $5 \mu \mathrm{m}$ ODS $4.6 \times 150 \mathrm{~mm}$ column at $32{ }^{\circ} \mathrm{C}$. The mobile phase was methanol-water (70:30) at a flow rate of $1.0 \mathrm{ml} / \mathrm{min}$ with an injection volume of $10 \mu \mathrm{l}$. The monitoring wavelength was $288 \mathrm{~nm}$.

Liquid chromatography - mass spectrometry

An Alliance 2690 separation module with auto sampler and quaternion gradient pump (Waters, USA.) was used for HPLC separation and Micromass ZMD4000 with ESI (negative mode) interface were used for mass spectrometry.

HPLC: The chromatographic column was a $2.1 \times 150 \mathrm{~mm}$ Symmetry C18 with $3.5 \mu \mathrm{m}$ size (Waters, USA.). The mobile phase was methanol (HPLC grade, Dikma) - re-deionized water (70:30) at a flow rate of $0.2 \mathrm{ml} / \mathrm{min}$, filtered with a Millipore $0.45 \mu \mathrm{m}$ filter before used. The injection volume was $10 \mu 1$.

ESI-MS: High purity nitrogen was used as the auxiliary gas. The operating parameters of MS were desolventized gas, $360 \mathrm{l} / \mathrm{h}$; cone gas, $104 \mathrm{l} / \mathrm{h}$; Cone voltage, $25 \mathrm{~V}$; capillary voltage, $3.5 \mathrm{kV}$; extractor voltage, $5 \mathrm{~V}$; source block temperature, $100^{\circ} \mathrm{C}$; and desolvation temperature, $250^{\circ} \mathrm{C}$. Mass spectral data were collected in the scan range of m/z from $100 \mathrm{amu}$ to $1000 \mathrm{amu}$. All LC-MS data were processed by MassLynx ${ }^{\mathrm{TM}}$ data acquisition software.

\section{Recovery}

Four groups of samples were injected to HPLC for detection. The first group of samples which did not add standard solution was blank control group. Detections of the other three groups of samples were compared with the blank, and then the recovery rate was obtained, listed in Table 1.

\section{Thermal stability}

Samples were placed in water bath for $2 \mathrm{~h}$ at $50{ }^{\circ} \mathrm{C}, 60^{\circ} \mathrm{C}, 70{ }^{\circ} \mathrm{C}$ and 80 ${ }^{\circ} \mathrm{C}$, respectively. After cooling down to $25^{\circ} \mathrm{C}$, they were diluted with ethanol to $25 \mathrm{ml}$ and then analyzed with HPLC. To observe the stability of kurarinone at room temperature, one group of samples were also analyzed after keeping at $25^{\circ} \mathrm{C}$ for $48 \mathrm{~h}$. Every group was repeated 2 times.

\section{RESULTS AND DISCUSSION}

Standard sample $\beta$ was identified with HPLC-ESI-MS, which started with a full scan (from m/z 100 to $\mathrm{m} / \mathrm{z} 1000$ ) of $1 \mu \mathrm{g} / \mathrm{ml}$ solutions of the powder in negative ion modes. A typical mass spectrum of standard kurarinone is shown in Fig. 1 (ESI: $\left.\mathrm{m} / \mathrm{z} 437.6[\mathrm{M}-\mathrm{H}]^{-}\right)$and the full scan of UV spectra is shown in Fig. 3. There are seven significant fragments in the spectra: $876.0,875.0,438.5$, 437.6, 275.3, 161.6 and 137.3 labeled in Fig. 1. The $\mathrm{m} / \mathrm{z}$ value at 876.0 and 875.0 were two dimeric-compounds formed in ESI experiment and $\mathrm{m} / \mathrm{z}$ value 438.5 was isotopic ion. The $\mathrm{m} / \mathrm{z} 437.6$ was quasi-molecular ion. The fractures mode of other three spectra: 275.3, 161.6, 137.3 are shown in Fig. 1 and the formulae of bond cleavages are consistent with Bai's work ${ }^{9}$. In published reference ${ }^{10}$, ESI-MS $^{2}$ was used, the $\mathrm{m} / \mathrm{z}$ values were $421\left(\left[M+\mathrm{H}_{-}-\mathrm{H}_{2} \mathrm{O}\right]^{+}\right), 315$ $\left([M+\mathrm{H}-\text { lanandulyl }]^{+}\right), 303\left({ }^{1,3} \mathrm{~A}^{+}-\mathrm{H}_{2} \mathrm{O}\right), 297\left(\left[M+\mathrm{H}-\text { lanandulyl }-\mathrm{H}_{2}^{2} \mathrm{O}\right]^{+}\right), 179$ $\left({ }^{1,3} \mathrm{~A}^{+}\right.$-lanandulyl) and group lanandulyl in his work might be lavanduly $l^{4,9,10}$. These results of $\mathrm{m} / \mathrm{z}$ values did not consistent with reference ${ }^{9}$ and the current work. The optimal UV detection wavelength was $288.5 \mathrm{~nm}$ according to Fig. 3, and UV of $288 \mathrm{~nm}$ was then adopted for HPLC detector in this work.

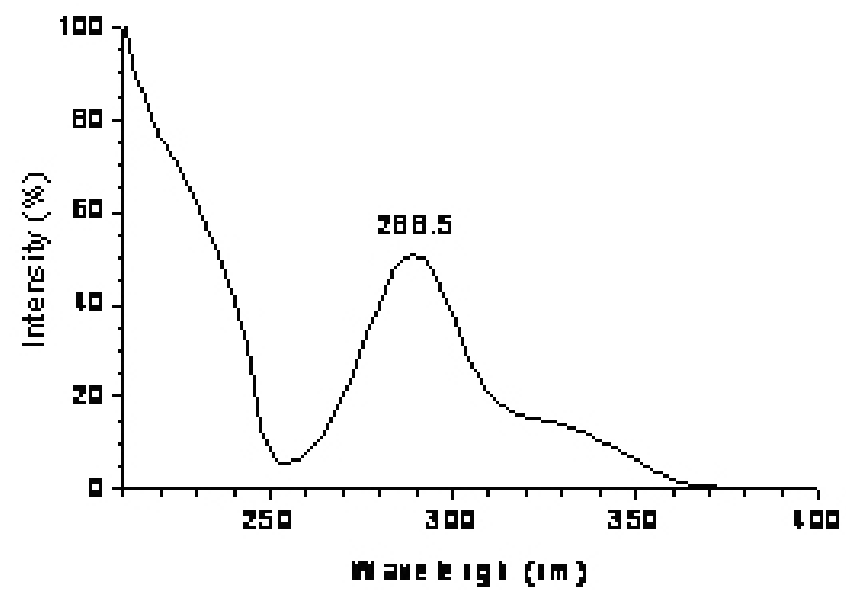

Fig. 3. Full scan of UV spectrum of kurarinone.

The chromatograms of standard sample $\beta$ are shown with the UV detection wavelength $288 \mathrm{~nm}, 270 \mathrm{~nm}$ and $320 \mathrm{~nm}$, respectively. In all spectra, the principal peak accounted for $95.5 \%$ of total peak area, as shown in Fig. 4. There are no obvious interfering peaks, which indicate that the purity of sample $\beta$ is almost $95.5 \%$. This proves that the process to obtain high purity kurarinone is feasible. 


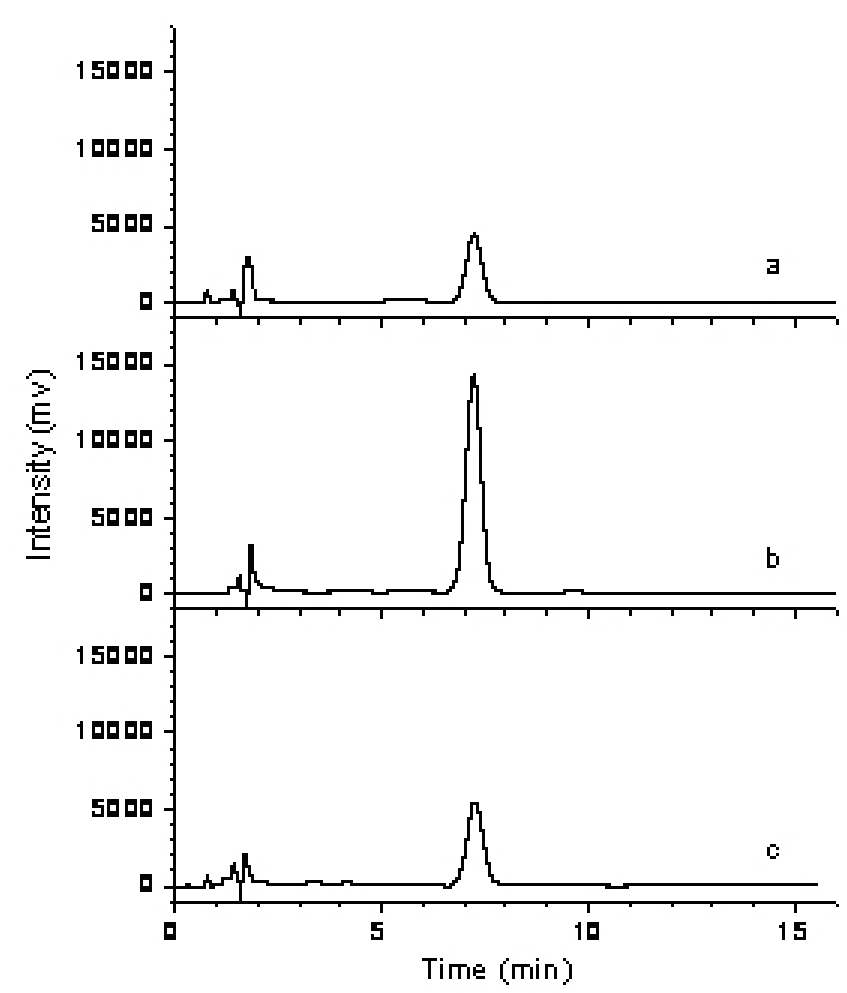

Fig. 4. HPLC chromatogram of kurarinone standard sample $(\beta)(4.56 \mu \mathrm{g} /$ ml) with UV detector at three different wavelengths of (a) $270 \mathrm{~nm}$, (b) $288 \mathrm{~nm}$, (c) $320 \mathrm{~nm}$.

There are no interfering peaks present near the principal peak of kurarinone, which implies the separation of kurarinone and other components was good. In this work, kurarinone was examined in linear ranges of $2.28-22.8 \mu \mathrm{g} / \mathrm{ml}$. The extractive recoveries of kurarinone were $98.7-102.9 \%$, RSD $2.09 \%$, as listed in Table 1. All of these show that the current HPLC method is effective in analysis of kurarinone.

Table 1. Extraction recovery of kurarinone.

\begin{tabular}{|c|r|r|r|}
\hline $\begin{array}{c}\text { Concentration of } \\
\text { added kurarinone } \\
(\mu \mathrm{g} / \mathrm{ml})\end{array}$ & $\begin{array}{c}\text { Observed } \\
\text { concentration } \\
(\mu \mathrm{g} / \mathrm{ml})\end{array}$ & Recovery (\%) & RSD (\%) \\
\hline 0 & 5.91 & - & - \\
\hline 2.28 & 8.16 & 98.7 & \\
\hline 4.56 & 10.67 & 104.4 & \multirow{2}{*}{2.09} \\
\hline 6.84 & 12.93 & 102.6 & \\
\hline
\end{tabular}

Every group was repeated 3 times, and mean value was adopted (RSD $<$ $4.31 \%)$. Recovery $=[$ (observed mass concentration - basal mass concentration $) /$ (mass concentration of added kurarinone) $] \times 100 \%$.

A comparison of extractive efficiencies among different treatments (ultrasonic extraction and maceration) is listed in Table 2. The relative extraction ratio of ultrasonic method is $81.4 \%$ versus that of maceration with $58.6 \%$, in the same period of $1 \mathrm{~h}$. The relative extraction of ultrasonic for 2 $\mathrm{h}$ reached $97.4 \%$; in contrast to that, it is only $85.7 \%$ by maceration for $6 \mathrm{~h}$ The relative recovery with maceration increased from $58.6 \%$ to $85.7 \%$ with the extraction time from $1 \mathrm{~h}$ to $6 \mathrm{~h}$. In contrast, ultrasonic extraction is not evident increase to prolong extraction time. High frequency ultrasonic wave propagation can destruct the pores so the solute can infiltrate in a short period of time ${ }^{7}$. All of these illustrate that ultrasonic extraction is much more effective than maceration in extracting of kurarinone in S. flavescens. The frontier one can improve extraction rates and avoid using heat.
Table 2. Extraction rates of kurarinone with different methods.

\begin{tabular}{|c|c|c|c|c|c|c|}
\hline \multirow{2}{*}{$\begin{array}{c}\text { Extracted } \\
\text { method }\end{array}$} & \multicolumn{4}{|c|}{ Recovery ratio $(\mathrm{mg} / \mathrm{g})$} & \multirow{2}{*}{$\begin{array}{c}\text { RSD } \\
(\%)\end{array}$} & $\begin{array}{c}\text { Relative } \\
\text { recovery } \\
\text { ratio (\%) }\end{array}$ \\
\cline { 2 - 5 } & 1 & 2 & 3 & Mean & & \\
\hline Ultrasonic $(1 \mathrm{~h})$ & 5.68 & 6.03 & 5.93 & 5.88 & 2.27 & 81.4 \\
\hline Ultrasonic $(2 \mathrm{~h})$ & 7.11 & 7.05 & 6.91 & 7.03 & 1.07 & 97.4 \\
\hline $\begin{array}{c}\text { Maceration } \\
(1 \mathrm{~h})\end{array}$ & 3.92 & 4.24 & 4.52 & 4.23 & 4.84 & 58.6 \\
\hline $\begin{array}{c}\text { Maceration } \\
(6 \mathrm{~h})\end{array}$ & 6.14 & 6.13 & 6.29 & 6.19 & 1.11 & 85.7 \\
\hline
\end{tabular}

The content of kurarinone in the dried powder was $7.22 \mathrm{mg} / \mathrm{g}$. Relative recovery ratio $=($ recovery ratio $/ 7.22) \times 100 \%$.

The thermal stability of kurarinone at different temperatures was examined, as shown in Table 3. All of the samples were in volumetric flask and placed in ultrasonic equipment to eliminate the air for $30 \mathrm{~min}$. Therefore, oxidation reaction of kurarinone was eliminated. It was reported that phumbagin had certain degradation when exposed at high temperature for a certain time ${ }^{6}$. Our results illustrate that kurarinone is stable at $25{ }^{\circ} \mathrm{C}$ at least for $48 \mathrm{~h}$. A very low decrease in the mass concentration of kurarinone was observed when the temperature is below $50{ }^{\circ} \mathrm{C}$ (holding for $2 \mathrm{~h}$ ). However, with increasing temperature up to $60^{\circ} \mathrm{C}$ and $70{ }^{\circ} \mathrm{C}$, the decreases in the mass concentration of kurarinone are evident reached $15.96 \%$ and $19.51 \%$. At $80^{\circ} \mathrm{C}$, the decrease ratio is even up to $25.54 \%$. The test of thermal stability illustrates that kurarinone should be conserved at a temperature below $25^{\circ} \mathrm{C}$.

Table 3. Decrease ratio of kurarinone at different temperatures

\begin{tabular}{|c|c|c|c|c|c|}
\hline Temperature $\left({ }^{\circ} \mathrm{C}\right)$ & 25 & 50 & 60 & 70 & 80 \\
\hline Duration $(\mathrm{h})$ & 48 & 2 & 2 & 2 & 2 \\
\hline $\begin{array}{c}\text { Mass concentration } \\
(\mu \mathrm{g} / \mathrm{ml})\end{array}$ & 20.5 & 20.0 & 17.2 & 16.6 & 15.3 \\
\hline Decrease ratio $(\%)$ & 0 & 2.6 & 16.0 & 19.2 & 25.5 \\
\hline
\end{tabular}

Data expressed as mean values with the $\mathrm{RSD}<3.28 \%(\mathrm{n}=3)$.

\section{CONCLUSIONS}

A herbal ingredient kurarinone (7, 2', 4'-trihydroxy-8-lavandulyl-5methoxy flavanone) was isolated from the root of $S$. flavescens. A reliable HPLC-MS method developed to determine kurarinone and the results are consistent with Bai's work ${ }^{9}$ but different with Zhang' ${ }^{10}$. Ultrasonic extraction is much more effective than maceration in extracting kurarinone from $S$. flavescens. The kurarinone sample with content of $95.5 \%$ was obtained by silica gel column followed by reverse-phase column. The thermal stability tests illustrate that kurarinone is stable below $25^{\circ} \mathrm{C}$, but a degradation of $25.54 \%$ is observed at $80^{\circ} \mathrm{C}$.

\section{ACKNOWLEDGEMENTS}

The authors are grateful for the financial supports of the National Natural Science Foundation of China (20590363), the Natural Science Foundation of Shanxi Province, and the State Key Fundamental Research Project of China.

\section{REFERENCES}

1. W. Tang, G. Eisenbrand, Chinese Drugs of Plant Origin, Springer-Verkag, New York, 1992.

2. X. Y. Zheng, et al, Chinese Pharmacopoeia, Chemical Industry Press, Beijing, 2005.

3. W. G. Ko, T. H. Kang, N. Y. Kim, S. J. Lee, Y. C. Kim, G. L. Ko, S. Y. Ryu, B. H. Lee, Toxicol in Vitro 14, 429, (2000).

4. S. W. Lee, H. S. Lee, J.Y. Nam, O. E. Kwon, J. A. Baek, J. S. Chang, M. C. Rho, Y.K. Kim, J. Ethnopharmacol. 97, 515, (2005).

5. S.J. Kim, K.H. Son,H.W. Chang, Biol. Pharm. Bull. 26, 1348, (2003).

6. S. R. D. Paiva, L. A. Lima, M. R. Figueiredo, M. A. C. Kaplan, An. Acad. Bras. Cienc. 76, 499, (2004).

7. M. Romdhane, C. Gourdon, Chem. Eng. J. 87, 11, (2002).

8. S. R. Sargenti, W. Vichnewski, Phytochem. Analysis 11, 69, (2000).

9. Y. Bai, M.Q. Guo, F. R. Song, Z. Q. Liu, S.Y. Liu, J. Chinese U. 25, 284, (2004).

10. L. Zhang, L. Xu, S. S. Xiao, Q. F. Liao, Q. Li, J. Liang, X. H. Chen, K. S. Bi, J. Pharmaceut. Biomed. 44, 1019, (2007). 